\title{
Kerr black holes and time profiles of gamma ray bursts
}

\author{
S. McBreen ${ }^{1}$, B. McBreen ${ }^{1}$, L. Hanlon ${ }^{1}$, and F. Quilligan ${ }^{1,2}$ \\ ${ }^{1}$ Department of Experimental Physics, University College Dublin, Dublin 4, Ireland \\ 2 Intel Corporation, Leixlip, Co. Kildare, Ireland
}

Received 11 June 2002 / Accepted 9 August 2002

\begin{abstract}
The cumulative light curves of Gamma Ray Bursts (GRBs) smooth the spiky nature of the running light curve. The cumulative count increases in an approximate linear way with time $t$ for most bursts. In 19 out of 398 GRBs with $T_{90}>2 \mathrm{~s}$, the cumulative light curve was found to increase with time as $\sim t^{2}$ implying a linear increase in the running light curve. The non-linear sections last for a substantial fraction of the GRB duration, have a large proportion of the cumulative count and many resolved pulses that usually end with the highest pulse in the burst. The reverse behaviour was found in 11 GRBs where the running light curve decreased with time and some bursts are good mirror images of the increases. These GRBs are among the spectrally hardest bursts observed by BATSE. The most likely interpretation is that these effects are signatures of black holes that are either being spun up or down in the accretion process. In the spin up case, the increasing Kerr parameter of the black hole allows additional rotational and accretion energy to become available for extraction. The process is reversed if the black hole is spun down by magnetic field torques. The luminosity changes in GRBs are consistent with the predictions of the BZ process and neutrino annihilation and thus provide the link to spinning black holes. GRBs provide a new window for studying the general relativistic effects of Kerr black holes.
\end{abstract}

Key words. gamma rays - bursts: gamma rays - observations: methods - data analysis: methods - statistical

\section{Introduction}

It is suspected that spinning black holes reside in a variety of astrophysical sources. Frame dragging creates a special region called the ergosphere in which any material or energy must rotate in the same direction as the black hole. The energetic reactions near the black hole maybe responsible for relativistic jets in active galactic nuclei (AGN), microquasars and gamma ray bursts (Koide et al. 2002; Frail et al. 2001). In GRBs the source of the enormous energy in gamma rays maybe the cataclysmic formation of a spinning black hole involving mergers of compact objects such as neutron star (NS) binaries or NS and black holes (Piran 1999; Ruffert \& Janka 1999) and also during or after the collapse of massive stars (MacFadyen \& Woosley 1999; Paczynski 1998; Vietri \& Stella 1998; Reeves et al. 2002). The central engine is hidden from view and only gravitational radiation and neutrinos may escape and reach the observer directly from the engine. A key feature of the internal shock model is that the observed gamma rays reflect the variability of the central engine and the GRB duration may be determined by the engine (Rees \& Mészáros 1994; Piran 1999). The cumulative output in gamma rays of a burst indirectly reflects the output of the central engine via a relativistic jet. The advantage of using the cumulative light curve is that it reveals the trends by smoothing the spiky nature of the

Send offprint requests to: $\mathrm{S}$. McBreen,

e-mail: smbreen@bermuda.ucd.ie running light curve. The cumulative light curves of most bursts can be approximated by a linear function of time and GRBs may be regarded as relaxation systems that continuously accumulate energy in the reservoir and discontinuously release it (McBreen et al. 2002a). In a relatively small number of GRBs, the cumulative light curves depart from linearity in a consistent way. The selection and properties of these GRBs are presented here in Sects. 2 and 3, discussed in Sect. 4 and summarised in Sect. 5 .

\section{Analysis of the light curves of gamma ray bursts}

A large sample of the brightest BATSE GRBs was used with the data combined from the four energy channels (Fishman \& Meegan 1995). The analysis procedures are described in detail elsewhere (Quilligan et al. 2002; McBreen et al. 2001, 2002a). The full sample consisted of 100 GRBs with duration $T_{90}<2 \mathrm{~s}$ where $T_{90}$ measures the burst integrated count from $5 \%$ to $95 \%$ of the total, 319 GRBs with $T_{90}>2 \mathrm{~s}$ and a further fainter sample of 79 GRBs with $T_{90}>100 \mathrm{~s}$ to include very long bursts. The cumulative light curves of most GRBs could be approximated by a linear function implying constant output over most of the duration of the burst (McBreen et al. 2002a). Two significant minorities were visually identified that are better described by nonlinear changes in the cumulative count. In category A the running count increased towards the tallest pulse in the burst resulting in a nonlinear increase in the 
cumulative profile. In category B the running count decreased after the tallest pulse in the burst causing the cumulative profile to increase at a much slower rate as time progressed. In category $\mathrm{A}$ the normalised cumulative profile was fit by the function

$R\left(t_{i}\right)=I_{\min }+c\left(t_{i}-t_{0}\right)^{\beta}$

where $R\left(t_{i}\right)$ is the cumulative count at time $t_{i}$ and $I_{\min }$ is the minimum count at $t_{0}$. In category $\mathrm{B}$ the function used was

$D\left(t_{i}\right)=I_{\max }-c\left(t_{\max }-t_{i}\right)^{\beta}$

where $D\left(t_{i}\right)$ is the cumulative count at time $t_{i}, t_{\max }$ is the time at the end of the fitted section of the burst and $I_{\max }$ is the value of the count at $t_{\max }$. $I_{\max }$ usually has a value close to unity. The profiles were fit using the Levenberg-Marquardt nonlinear minimisation algorithm. The fitted sections include at least 5 pulses $\geq 5 \sigma$ from the running profile, $20 \%$ of the GRB duration and $30 \%$ of the cumulative total. In the two categories there were 19 and 11 GRBs that satisfied the selection criteria. An increase in number occurs when the selection criteria are relaxed.

The median number of pulses $N$ in GRBs with $T_{90}>2 \mathrm{~s}$ is only 6 (Quilligan et al. 2002) and the requirement on $N$ restricts the GRBs to about half of the total. The number of pulses is important because they may originate from explosions in the central engine and discriminate against GRBs, where the emission is not well resolved into pulses because of the washing out of time structure in the jet before the gamma-ray photosphere, and interactions with the external medium including the effects of early afterglow (Dermer \& Mitman 1999). The changes in the cumulative count presented here are quite different from the smooth power law decays in GRBs of the FRED (i.e. Fast Rise Exponential Decay) type (Giblin et al. 2002) and the smooth decays in a sample of pulses within GRBs (Ryde \& Svensson 2001).

Most GRBs with $T_{90}<2 \mathrm{~s}$ can also be approximated by a linear fit to the cumulative light curve (McBreen et al. 2002a). The GRBs have a median value of $N=2.5$ (McBreen et al. 2001) and no short GRBs were found to meet the criteria used for long GRBs.

\section{Results on the light curves of GRBs}

The 19/11 GRBs that satisfied the selection for categories A/B are listed in Table 1. The running and cumulative light curves and fits to the nonlinear sections of the bursts are given in Figs. 1 and 2 for three bursts in each category. The cumulative light curve smoothes the spiky nature of the running light curve. In category A the highest peak flux frequently occurs near the end of the nonlinear increase and subsequently the bursts stops abruptly (Fig. 1a) or continues at a much reduced rate (Fig. 1c) due to decrease in accretion. In category $B$ there is a decrease in the running light curve that is an approximate mirror image of bursts in category A (Fig. 2) and the nonlinear slowdown in the cumulative light curve results from the drop in amplitude of the pulses in the running profiles.
The GRBs in Table 1 have large numbers of pulses. The errors due to the BATSE counting statistics can be neglected because they are very small compared with the large fluctuations caused by the pulses in the running light curves. Equations (1) and (2) were fit to the cumulative light curve to obtain the best fit to the data (Figs. 1 and 2). The values of $\beta$ are in the range 1.8 to 2.3 (Table 1 ). The range in $\beta$ was estimated by varying $\beta$ from 1.5 to 2.5 and visually estimating the range where there was agreement between the fitted functions and the cumulative light curves. The values are listed in Table 1 and typically are in the range \pm 0.2 . The fluctuations between the fitted curve and the cumulative data are due to the pulses and the time intervals between them in the running light curve. These residuals are inherent to the pulsed nature of GRBs. The value of $c$ (Table 1) is given for the parabolic value $\beta=2$ for all bursts to enable comparison of the normalised cumulative profiles. The errors on the hardness ratios (Table 1) are all less than $7 \%$ with the exception of the soft GRB 6903 where it is $50 \%$.

\section{Discussion}

The nonlinear changes in the light curves are usually evident in all four BATSE channels. The achromatic nature of the signals seem to exclude an effect due to changes in the optical depth. It has been suggested that some of the time structure in GRB light curves could be due to precession of the black hole equator and the accretion disk (Portegies Zwart et al. 1999; Fargion 1999; Romero et al. 1999; Thorne et al. 1986; MacFadyen \& Woosley 1999). The estimated precessional period is about $1 \mathrm{~s}$ and is too small to account for the gradual increases and decreases in the GRB light curves. The nonlinearities in the light curves could be a subtle signature of the central engine that only occur in some GRBs. Most models of GRBs favour a hyper-accretion process into a newly formed black hole and in these systems the nonlinearities might be due to systematic changes in the accretion rate or to general relativistic effects of a spinning black hole. All the evolutionary scenarios, such as mergers of compact objects and collapsars, create rapidly rotating black holes because they accrete a fraction of their mass from a disk. However the engine must be capable of generating GRBs with many pulses with progressively increasing amplitudes and GRBs that appear to be their mirror images with harder spectra.

When a black hole swallows matter and radiation from an accretion disk, its mass and angular momentum evolve. The evolution of the black hole was first evaluated by Bardeen (1970) following the initial work of Lynden-Bell (1969). The spin of the black hole, as measured by the Kerr parameter $a=J c / G M^{2}$ where $M$ is the mass of the black hole and $J$ its angular momentum, increases nonlinearily with accretion from 0 to approach but not equal 1 for an extreme Kerr black hole (Thorne 1974). The equatorial radius of the ergosphere decreases from $r_{\mathrm{e}}=2 G M / c^{2}$ to $G M / c^{2}$ and the radius of the marginally stable orbit also decreases from $r_{\mathrm{ms}}=6 \mathrm{GM} / \mathrm{c}^{2}$ to $G M / c^{2}$ and additional gravitational potential energy becomes available. The dependance of $a$ on accreted mass is given by Thorne (1974) and plotted in Popham et al. (1999). Substantial accretion is required to significantly alter the value 

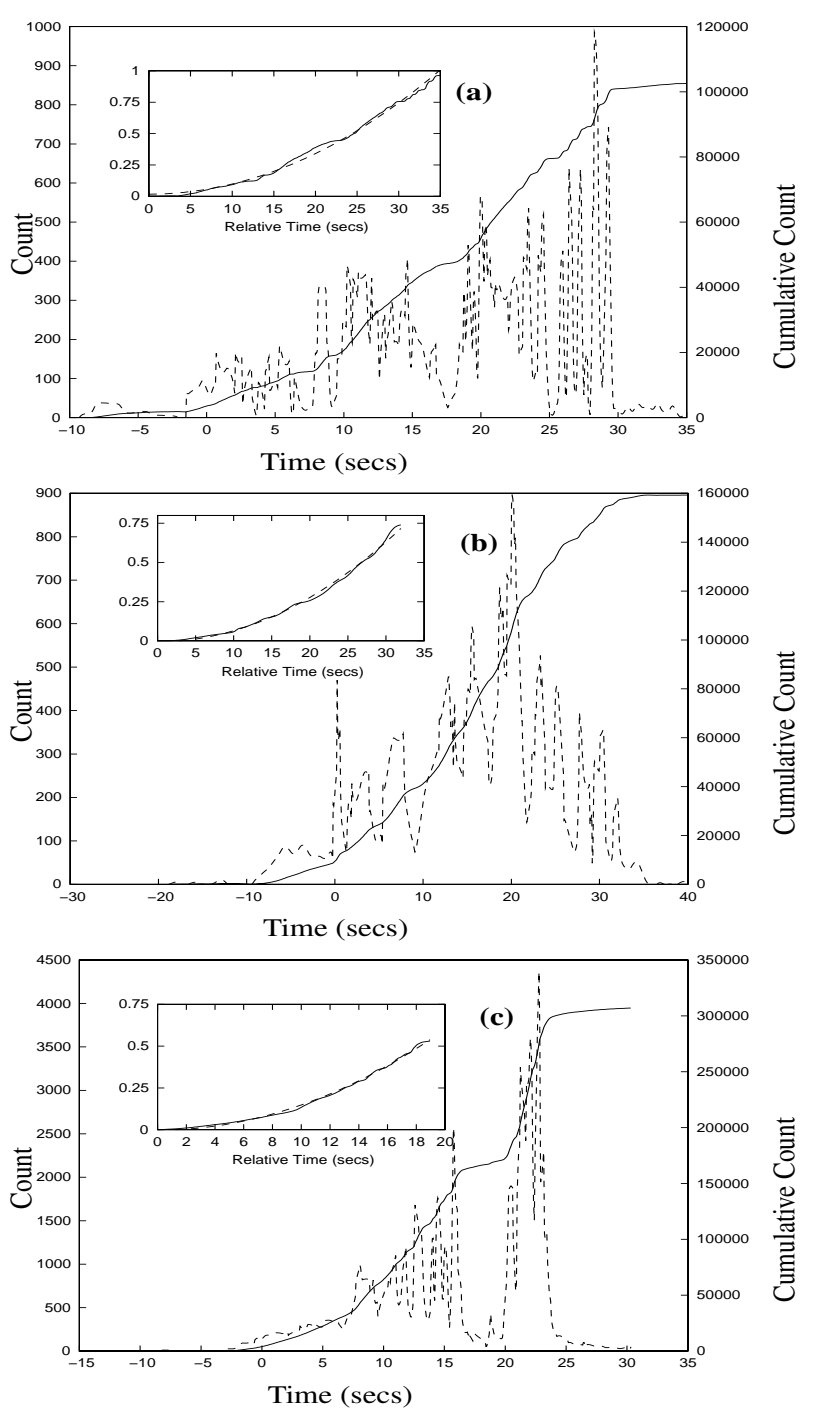

Fig. 1. The running (dashed) and cumulative (solid) light curves of the BATSE bursts with trigger numbers a) 3105, b) 3860 and c) 6963 with count per $64 \mathrm{~ms}$ and cumulative count scales on the left and right vertical axes. The inserts give the cumulative count (solid) and the fit of the function (dashed) for the relevent section. The start and end times are listed in Table 1 and $t_{1}$ is shifted to zero for the inserts. The vertical axes in the inserts are the normalised cumulative count.

of $a$ which acquires a value $a \sim 0.97$ when the mass of the hole has been accreted. Many models of GRBs require extremely high accretion rates and for a typical long duration GRB could be about $0.1 M_{\odot} \mathrm{s}^{-1}$. There could be substantial changes in $a$ during a GRB and it is necessary to know how the power output varies with $a$ and time. Assuming a constant mass accretion rate $a$ evolves with time as $\sim t^{0.5}$ for $0.3<a<0.8$ and more slowly/rapidly for $a>0.8 / a<0.3$ (Popham et al. 1999).

There are three energy extraction processes to consider and precise predictions are not always available because of the complexity of these systems (Mészáros 2000; Rees 1999). In the standard accretion process, the viscous energy dissipation is balanced by neutrino cooling. Popham et al. (1999) called it neutrino dominated accretion flow (NDAF) and worked out its properties including variations in the disk mass, accretion rate, black hole mass and Kerr parameter. The properties of
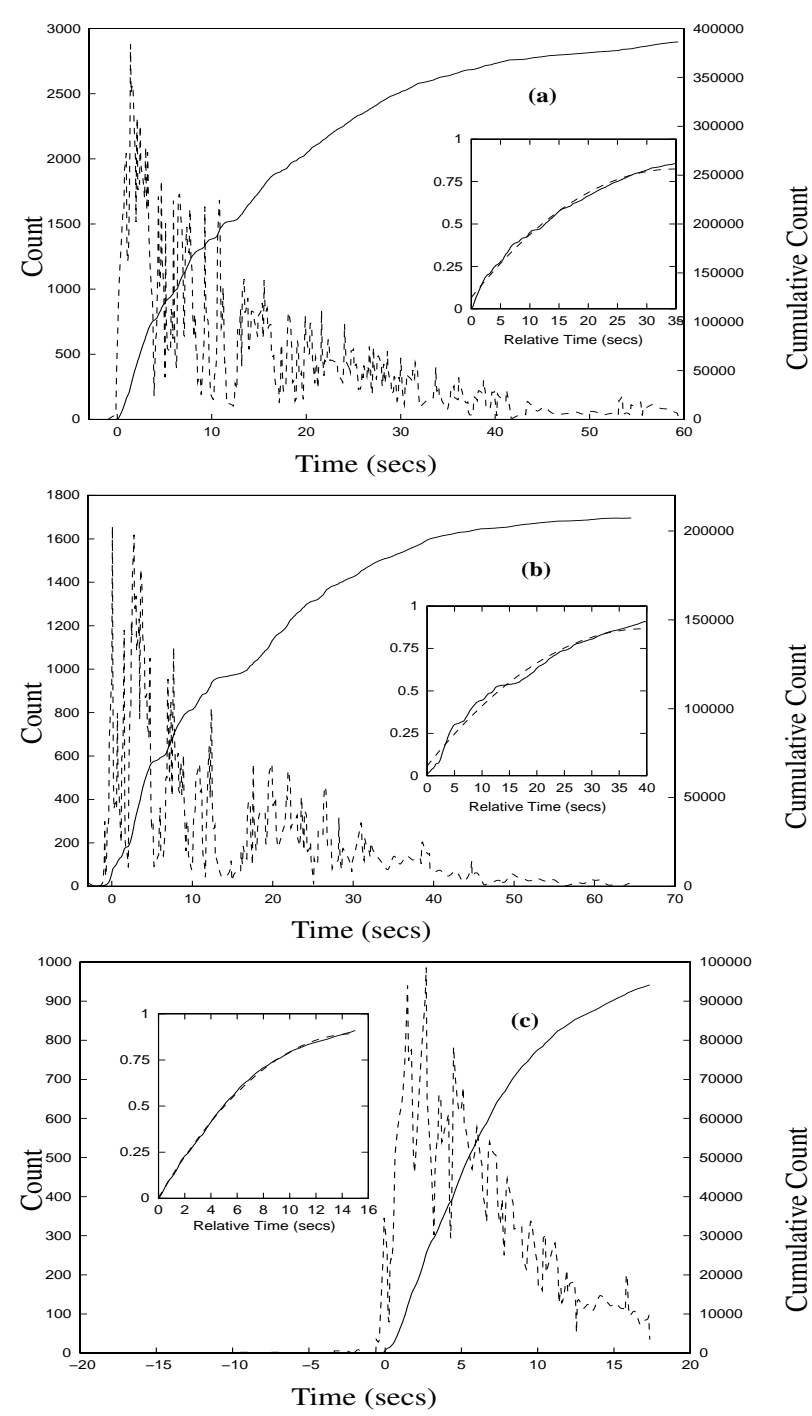

Fig. 2. The running (dashed) and cumulative (solid) light curves of the BATSE bursts with trigger numbers a) 678, b) 4039 and c) 6694 with the same notation as Fig. 1.

these disks have been further explored (Narayan et al. 2001). One extraction mechanism is the $v \bar{v} \rightarrow \mathrm{e}^{+} \mathrm{e}^{-}$process that taps the thermal energy of the disk. The annihilation of neutrinos close to the axis of a rotating system, where there is a low concentration of baryons, was proposed as a way to produce a clean fireball (Eichler et al. 1989; Mészáros \& Rees 1992). A number of models have been used to estimate the neutrino emission from NS binaries and NS and black holes (Ruffert \& Janka 1999; Lee 2000; Janka et al. 1999; Rosswog \& Davies 2002). In a more complete model (Popham et al. 1999) the neutrino luminosity and energy deposition in the polar regions was numerically evaluated using the Kerr metric. The integrated energy deposited by $v \bar{v} \rightarrow \mathrm{e}^{+} \mathrm{e}^{-}$increases with time as $\sim t^{2}$ when $a$ increases above an initial value of 0.5 (Figs. 21 and 22 in MacFadyen \& Woosley 1999) and including neutrinos down to the last stable orbit. The calculations reveal that at higher accretion rates the neutrino luminosity and efficiency for annihilation increase. These parameters also increase with the Kerr parameter $a$. As $a$ increases the last stable orbit moves inward. The neutrino emission from the higher gravitational 
Table 1. The GRBs that satisfied the criteria for category A (first 19 rows) and category $\mathrm{B}$ (second 11 rows). The columns refer to BATSE trigger number, the number of pulses $N \geq 5 \sigma$, the number of those pulses $N^{\prime}$ included in the fitted region between times $t_{1}$ and $t_{2}$ with a minimum value of $7 \mathrm{~s}$ for GRB 1440 and maximum of $140 \mathrm{~s}$ for GRB 8101, the hardness ratio (HR) which is the ratio of fluence in BATSE channels 3 and 4 above $100 \mathrm{keV}$ to that in channels 1 and 2 below $100 \mathrm{keV}$, the percentage of the total integrated counts $(\% \mathrm{C})$ in the fitted region, the index $\beta$ and the coefficient $c \times 10^{-4}$ for the fit with $\beta=2$. GRBs 3247, 6353, 6903, 8101 and 7660 are from the fainter sample with $T_{90}>100 \mathrm{~s}$.

\begin{tabular}{|c|c|c|c|c|c|c|}
\hline Burst & $N / N^{\prime}$ & $t_{1}, t_{2}$ & HR & $\% \mathrm{C}$ & $\beta$ & $c$ \\
\hline 394 & $25 / 12$ & 0,25 & 4.6 & 42 & $1.9 \pm 0.2$ & 6.8 \\
\hline 1122 & $10 / 8$ & 0,11 & 3.2 & 62 & $1.9 \pm 0.2$ & 49.0 \\
\hline 1440 & $15 / 9$ & 10,17 & 5.3 & 70 & $2.1 \pm 0.2$ & 147.1 \\
\hline 2450 & $22 / 11$ & 45,65 & 3.4 & 49 & $2.1 \pm 0.3$ & 12.0 \\
\hline 3035 & $21 / 12$ & 5,75 & 4.7 & 52 & $2.1 \pm 0.3$ & 1.1 \\
\hline 3105 & $30 / 30$ & $-10,30$ & 6.5 & 98 & $2.1 \pm 0.2$ & 6.3 \\
\hline 3247 & $25 / 17$ & 80,180 & 7.7 & 79 & $2.1 \pm 0.2$ & 0.9 \\
\hline 3489 & $12 / 5$ & $-2,11$ & 6.6 & 56 & $2.0 \pm 0.2$ & 35.4 \\
\hline 3860 & $13 / 8$ & $-5,22$ & 22.4 & 72 & $1.9 \pm 0.3$ & 9.6 \\
\hline 5526 & $34 / 14$ & $-6,16$ & 4.4 & 36 & $2.1 \pm 0.2$ & 7.6 \\
\hline 6353 & $9 / 5$ & $-10,50$ & 1.7 & 53 & $2.2 \pm 0.2$ & 1.5 \\
\hline 6453 & $25 / 12$ & $-5,52$ & 1.7 & 46 & $2.0 \pm 0.3$ & 1.4 \\
\hline 6587 & $28 / 20$ & 3,25 & 7.6 & 73 & $2.1 \pm 0.2$ & 15.8 \\
\hline 6593 & $20 / 11$ & $-10,14$ & 4.7 & 60 & $2.1 \pm 0.3$ & 10.2 \\
\hline 6903 & $13 / 5$ & $-25,15$ & 1.6 & 47 & $2.1 \pm 0.2$ & 3.3 \\
\hline 6963 & $17 / 10$ & 0,17 & 4.1 & 52 & $2.0 \pm 0.2$ & 18.4 \\
\hline 7318 & $12 / 7$ & $-3,10$ & 21.8 & 78 & $2.0 \pm 0.2$ & 48.0 \\
\hline 7575 & $30 / 20$ & 135,165 & 12.9 & 78 & $2.1 \pm 0.2$ & 9.1 \\
\hline 8101 & $11 / 9$ & $-40,100$ & 10.4 & 95 & $1.9 \pm 0.2$ & 0.5 \\
\hline 678 & $52 / 44$ & 1,36 & 42.6 & 86 & $1.9 \pm 0.2$ & 6.2 \\
\hline 2891 & $18 / 14$ & $0.5,20$ & 23.1 & $892.1 \pm 0.3$ & 22.0 & \\
\hline 2929 & $44 / 36$ & 12,50 & 13.5 & 83 & $2.2 \pm 0.3$ & 5.7 \\
\hline 2984 & $22 / 12$ & 6,20 & 14.2 & 72 & $2.1 \pm 0.2$ & 37.2 \\
\hline 2993 & $17 / 11$ & 0,20 & 31.1 & 75 & $2.2 \pm 0.2$ & 16.4 \\
\hline 2994 & $36 / 22$ & 6,35 & 19.3 & 58 & $2.3 \pm 0.2$ & 6.3 \\
\hline 3408 & $44 / 35$ & 10,60 & 6.4 & 77 & $1.9 \pm 0.2$ & 3.2 \\
\hline 4039 & $33 / 31$ & 0,40 & 21.5 & 91 & $1.9 \pm 0.3$ & 5.1 \\
\hline 6694 & $10 / 9$ & 1,16 & 18.3 & 91 & $2.1 \pm 0.2$ & 39.5 \\
\hline 7660 & $7 / 5$ & 25,140 & 8.4 & 77 & $2.0 \pm 0.3$ & 0.7 \\
\hline 7766 & $22 / 11$ & 0,20 & 48.2 & 85 & $2.0 \pm 0.2$ & 18.1 \\
\hline
\end{tabular}

potential increases both the luminosity and temperature and also increases the density of neutrinos because of the more compact geometry (Asano \& Fukuyama 2001; Salmonson \& Wilson 1999). The model predictions are in good agreement with the cumulative light curves presented here. However the annihilation rate scales as the square of the neutrino luminosity (Mészáros 2000) and the efficiency is low if the production is too gradual.

An alternative and more efficient process for extracting energy maybe dissipation of magnetic fields generated by differential rotation in the disk. This extraction process is largely independent of $a$ and includes a relativistic wind or jet from the disk and flares (e.g. Kluźniak \& Ruderman 1998; Rees 1999; Livio et al. 1999). However a large energy source is also available in the spin of the black hole that maybe extracted by MHD coupling in the Blandford-Znajek (BZ) process (Blandford \& Znajek 1977) and the MHD Penrose process (Koide et al. 2002). The rotational energy available is $0.29 M c^{2}$ for a maximally rotating black hole. The BZ process behaves as an interaction of the black hole with its surrounding electromagnetic field and depends directly on the spin of the black hole. The predicted luminosity is $L \sim$ $10^{50} a^{2}\left(M / 3 M_{\odot}\right)^{2}\left(B / 10^{15} G\right)^{2} \operatorname{ergs~s}^{-1}$ where the magnetic field is supplied by the torus. It is interesting that $L \propto a^{2} \propto t$ provided $B$ remains constant on the horizon. This process also predicts a cumulative luminosity that increases $\sim t^{2}$. Furthermore there are the GRBs with many pulses with progressively declining amplitudes (Fig. 2 and Table 1) and running light curves that are reasonable mirror images of the increases in output. These GRBs appear to be good examples of the reverse process, namely, the decrease in the Kerr parameter $a$ produced by the slowing down of rapidly spinning black holes by the BZ or other processes (van Putten 2001; Lee \& Kim 2002; Li 2002). The BZ process can account for the behaviour of the cumulative light curves of GRBs by spin-up and spin-down of the black hole.

There are significant spectral differences between the bursts in Table 1 with the median values of the HRs of 5.3 and 19.3 for the two categories. The remainder of the GRBs with $T_{90}>2 \mathrm{~s}$ have median values of the HR of 4.1. The corresponding median values of the peak energies $E_{\text {peak }}$ for available GRBs are $340 \mathrm{keV}$ and $956 \mathrm{keV}$ for categories A and B (LloydRonning \& Ramirez-Ruiz 2002). The GRBs in category B are among the spectrally hardest BATSE GRBs, with the value of $E_{\text {peak }}$ generally following the intensity during the burst (Preece et al. 2000). The property of the central engine that maybe responsible for this behaviour is the spin of the black hole. The BZ mechanism operates most efficiently for a spun up black hole that is threaded by a powerful magnetic field. It appears that this system will generate more energetic outbursts with spectrally harder GRBs and observed range of pulse properties (e.g. Norris 2002; McBreen et al. 2002a; Lee et al. 2000b; Ramirez-Ruiz \& Fenimore 2000; Quilligan et al. 2002; Nakar \& Piran 2002). The progenitor systems should have high angular momentum and also form smaller mass black holes that are spun up and down more easily. The high spin of the black hole and angular momentum increasing outwards can stabilise the disk against runaway radial instabilities (Daigne \& Mochkovitch 1998).

The majority of GRBs with $T_{90}>2$ s have cumulative light curves that increase in a linear way with $t$. They do not have substantial nonlinear behaviour that meet the stringent criteria adopted here. There are a range of possibilities to account for this steady state behaviour including variations in the accretion rate or magnetic field that mask the slow changes due to $a$, different progenitors and disk emission dominating neutrino annihilation and the $\mathrm{BZ}$ process. In the later case the majority of GRBs are powered by emission from the accretion disk. However the relative contribution from MHD processes in the disk and spinning black hole are a matter of debate (Lee et al. 2000a).

In a few cases there is a gap in the light curves of the nonlinear increases and decreases where the output appears to 
be suspended for a short interval (e.g. Fig. 1c) and BATSE trigger numbers 2929, 3408 and 8101. The time intervals between pulses can be accommodated in models of GRBs as relaxation systems (Ramirez-Ruiz \& Merloni 2001; McBreen et al. 2002a). GRBs with $T_{90}<2 \mathrm{~s}$ are well described by linear increases in the cumulative counts and no convincing cases were found with nonlinear behaviour perhaps because of the short duration of the emission and small number of pulses.

\section{Conclusions}

The results of this study of BATSE light curve of GRBs are presented. The analysis revealed that the running light curve of a minority of bursts increase or decrease approximately linearly with time. These results are interpreted as possible evidence for spin-up and spin-down of black holes during the burst. The $\mathrm{BZ}$ process and neutrino annihilation are consistent with these results. The brute force appearance of Kerr black holes being spun up an down by hyperaccretion in GRBs contrasts with the more sedate and longer lasting indications of black holes in MCG-6-30-15 and other sources (Wilms et al. 2001; Miller et al. 2002; Woo \& Urry 2002; Koide et al. 2002).

\section{References}

Asano, K., \& Fukuyama, T. 2001, ApJ, 546, 1019

Bardeen, J. M. 1970, Nature, 226, 64

Blandford, R. D., \& Znajek, R. L. 1977, MNRAS, 179, 433

Daigne, F., \& Mochkovitch, R. 1998, MNRAS, 296, 275

Dermer, C. D., \& Mitman, K. E. 1999, ApJ, 513, L5

Eichler, D., Livio, M., Piran, T., \& Schramm, D. 1989, Nature, 340, 126

Fargion, D. 1999, A\&AS, 138, 507

Fishman, G. J., \& Meegan, C. A. 1995, Ann. Rev. Astron. Ap., 33, 415

Frail, D. A., Kulkarni, S. R., Sari, R., et al. 2001, ApJ, 562, L55

Giblin, T. W., Connaughton, V., van Paradijs, J., et al. 2002, ApJ, 570, 573

Janka, H.-T., Eberl, T., Ruffert, M., \& Fryer, C. L. 1999, ApJ, 527, 39

Kluźniak, W., \& Ruderman, M. 1998, ApJ, 508, L113

Koide, S., Shibata, K., Kudoh, T., \& Meier, D. L. 2002, Science, 295, 1688

Lee, W. H. 2000, MNRAS, 318, 606

Lee, H. K., Wijers, R. A. M. J., \& Brown, G. E. 2000a, Phys. Rep., 325,83
Lee, A., Bloom, E. D., \& Petrosian, V. 2000b, ApJS, 131, 21

Lee, H. K., \& Kim, H. E. 2002 [astro-ph/0201263]

Li, Li-Xin. 2002, ApJ, 567, 463

Livio, M., Ogilvie, G., \& Pringle, J. 1999, ApJ, 512, 100

Lloyd-Ronning, N. M., \& Ramirez-Ruiz, E. 2002 [astro-ph/0205127]

Lynden-Bell, D. 1969, Nature, 223, 690

MacFadyen, A. I., \& Woosley, S. E. 1999, ApJ, 524, 262

McBreen, S., Quilligan, F., McBreen, B., Hanlon, L., \& Watson, D. 2001, A\&A, 380, L31

McBreen, S., McBreen, B., Hanlon, L., \& Quilligan, F. 2002a, A\&A, in press

McBreen, S., McBreen, B., Quilligan, F., \& Hanlon, L. 2002b, A\&A, 385, L19

Mészáros, P. 2000, Nucl. Phys. B., 80, 63

Mészáros, P., \& Rees, M. J. 1992, MNRAS, 257, 29

Miller, J. M., Fabian, A. C., \& Wijnands, R. 2002 [astro-ph/0202375]

Nakar, E., \& Piran, T. 2002, ApJ, 572, L139

Narayan, R., Piran, T., \& Kumar, P. 2001, ApJ, 557, 949

Norris, J. P. 2002 [astro-ph/0201503]

Paczynski, B. 1998, ApJ, 494, L45

Piran, T. 1999, Phys. Rep., 314, 575

Popham, R., Woosley, S. E., \& Fryer, C. 1999, ApJ, 518, 356

Portegies Zwart, S. F., Lee, C., \& Lee, H. K. 1999, ApJ, 520, 666

Preece, R. D., Briggs, M. S., Mallozzi, R. S. et al. 2000, ApJS, 126, 19

Quilligan, F., McBreen, B., Hanlon, L., et al. 2002, A\&A, 385, 377

Ramirez-Ruiz, E., \& Fenimore, E. E. 2000, ApJ, 539, 712

Ramirez-Ruiz, E., \& Merloni, A. 2001, MNRAS, 320, L25

Rees, M. J. 1999, A\&AS, 138, 491

Rees, M. J., \& Mészáros, P. 1994, ApJ, 430, L93

Reeves, J. N., Watson, D., Osborne, J. P., et al. 2002, Nature, 416, 512

Romero, G. E., Torres, D., Andruchow, I., Anchordoqui, L., \& Link, B. 1999, MNRAS, 308, 799

Rosswog, S., \& Davies, M. B. 2002, MNRAS, 334, 481

Ruffert, M., \& Janka, H. T. 1999, A\&A, 344, 573

Ryde, F., \& Svensson, R. 2001 [astro-ph/0110196]

Salmonson, J. D., \& Wilson, J. R. 1999, ApJ, 517, 859

Thorne, K. S. 1974, ApJ, 191, 507

Thorne, K. S., Price, R. H., \& MacDonald, D. A. (eds.) 1986, Black holes: The Membrane Paradigm (Yale University Press)

van Putten, M. H. P. M. 2001, Phys. Rep., 345, 1

Vietri, M., \& Stella, L. 1998, ApJ, 507, L45

Wilms, J., Reynolds, C. S., Begelman, M. C. et al. 2001, MNRAS, 328, L27

Woo, J-H., \& Urry, P. M. 2002 [astro-ph/0207249] 